\title{
Spike development inhibition in the ftin mutant is associated with multiple phenotypic characteristics and regulated multiple biological pathways
}

\author{
Yong-sheng Zheng ${ }^{1}$, Jinpeng Zhang ${ }^{1}$, Cheng $\mathrm{Liu}^{2}$, Han Zhang ${ }^{2}$, Xiajie Ji ${ }^{1}$, Mumu Wang ${ }^{2}$, \\ Hui Wang ${ }^{2}$, Rongzhi Zhang ${ }^{2}$, Ruyu Li ${ }^{2}$, and Weihua Liu ${ }^{1}$ \\ ${ }^{1}$ Chinese Academy of Agricultural Sciences \\ ${ }^{2}$ Shandong Academy of Agricultural Sciences
}

July 31,2020

\begin{abstract}
Spike development of wheat line 3558M was strongly inhibited by low temperature stress in spring. The fertile tiller inhibition (ftin) gene in the wheat line $3558 \mathrm{M}$ is associated with multiple phenotypes, including the production of fewer tillers, delayed floral transition, and death of the shoot apical meristem. In order to study the relationship between spike development inhibition and response to cold stress, we systematically investigated the genes and pathways underlying the differences using ITRAQ proteomics and RNA-sequencing technologies. The results showed that the cold acclimation pathway and multiple defence responses, including reactive oxygen species-mediated hypersensitive response, salicylic acid-mediated systemic acquired resistance, are activated and led to apex death of the wheat line 3558M under cold stress. Meanwhile, the cold acclimation pathway inhibited the SVP-SCO1-LFY flowering pathway and led to delayed floral transition. Two TaPIN proteins were significantly downregulated, and multiple auxin signalling genes were also differentially expressed. Particularly, knocking down the two TaPIN genes using RNAi technology significantly reduced the tiller number. The cold stress might disrupt the distribution of auxin and reduce the tillers of 3558M. Taken together, the ftin gene might be a cold-sensitive mutation and that is the cause of multiple biological pathways and phenotypic changes.
\end{abstract}

\section{Hosted file}

spike development inhibition in the ftin mutant is associated with multiple phenotypic characteristics available at https://authorea.com/users/313917/articles/473430-spike-development-inhibitionin-the-ftin-mutant-is-associated-with-multiple-phenotypic-characteristics-and-regulatedmultiple-biological-pathways 